\title{
Reciclagem de papel: uma experiência de ensino, extensão e pesquisa
}

Paper recycling: a learning, extension and research experience

\section{Introdução}

As questões sobre a proteção ambiental têm sido foco de debates, de eventos científicos e de estudos em diversos níveis acadêmicos abordando temáticas variadas. Atualmente, uma das preocupações existentes é minimizar os danos gerados pelos acúmulos de resíduos sólidos, bem como os danos referentes à quantidade desses resíduos lançados no meio ambiente. Isso tem gerado reflexões que atravessam as fronteiras entre os países e continentes ${ }^{1}$.

Segundo Mattar ${ }^{2}$, o Presidente do Instituto Akatu refere que vivemos uma situação de enorme gravidade se considerarmos que, no modelo atual de consumo e de produção, já consumimos mais do que a capacidade de renovação dos recursos naturais. Nós estamos consumindo $20 \%$ a mais do que a Terra consegue sustentar.

A elaboração de propostas de um crescimento baseado em sustentabilidade torna-se cada vez mais forte e presente em nossas vidas e principalmente no mundo cooperativo; com isso, percebe-se que a humanidade está reconhecendo sua responsabilidade diante das condutas que vêm constantemente sendo lesivas ao meio ambiente ${ }^{3}$.

Atualmente, Mattar ${ }^{2}$ afirma que 20\% da população mundial realizam aproximadamente $86 \%$ do total de compras que são feitas no mundo. Um relatório das Nações Unidas de 1998 revela que esses $20 \%$ da população, dentre outras coisas que consome, é responsável pelo consumo de $85 \%$ do papel.

Por isso, o enfrentamento de problemas gerados pelos acúmulos de resíduos no meio ambiente tem sido a ordem do dia na mesa de debates por diversos setores sociais, entidades e organizações, devido à sua dimensão, complexidade e necessi-
Luciana Guimarães Assad', Lina Márcia Miguéis Beradinelli², Dalila Passos Pereira da Silva ${ }^{3}$, Antonio Augusto Braga de Oliveira Junior ${ }^{4}$ e Thamires Goldim Rodrigues $^{5}$

\section{Resumo}

Relato de experiência oriundo do projeto de extensão "A Reciclagem na coleta de papel na Faculdade de Enfermagem e no Hospital Universitário Pedro Ernesto (HUPE): uma prática de ensino, extensão e pesquisa". O objetivo é relatar a experiência de implantação da coleta seletiva de resíduos sólidos nos setores administrativos do HUPE e apresentar os resultados de uma pesquisa descritiva, quantitativa, desenvolvida em 2010 no HUPE. Aplicaram-se 162 questionários com os funcionários técnico-administrativos do HUPE, em 18 unidades. Foram respondidos 135 questionários cujos dados foram organizados e tabulados em frequência simples e percentual. Os resultados demonstram que a coleta de resíduos sólidos para reciclagem é considerada muito importante para $83 \%$ dos participantes. O projeto está implantado no HUPE em 18 setores administrativos e será implantado também em 29 salas da Faculdade de Enfermagem da UERJ. As ações do projeto têm contribuído para a formação de multiplicadores junto à comunidade e, principalmente, trazem para a reflexão diária a necessidade de redução, reutilização e reciclagem dos resíduos produzidos diariamente nas unidades parceiras.

Palavras-chaves: Resíduos Sólidos; Educação Ambiental; Reciclagem

Área Temática: Meio Ambiente Linha de Extensão: Questões Ambientais

Professora Adjunta. UERJ. E-mail: Igassad@terra.com.br 2 Professora Adjunta. UERJ. E-mail: I.m.b@uol.com.br 3 Bioquímica. UERJ. E-mail: dalila@uerj.br

${ }^{4}$ Tecnólogo. UERJ. E-mail: aabojr@hotmail.com

${ }^{5}$ Bolsista de Extensão. UERJ. E-mail: thamiresgondim@hotmail.com 
dade de investimentos pesados. Estes envolvem tecnologias que promovam o tratamento ou recuperação de resíduos, e também verbas necessárias à destinação adequada desses resíduos, bem como os investimentos relativos aos custos necessários para que se cumpram estratégias de sensibilização da sociedade com vistas a que ela seja mobilizada a esse favor ${ }^{4}$.

Trigueiro ${ }^{5}$ refere que os resíduos sólidos assim como o saneamento básico não recebem a devida atenção do poder público. O problema é grave e não é novo. Certamente também não é de simples equacionamento, mas sua complexidade e os custos envolvidos não podem permitir que em vez de ações efetivas os responsáveis continuem varrendo a sujeira para baixo do tapete.

Nesse sentido, considerando o quadro acima, e, em função das duas Unidades, cenários da pesquisa, possuírem uma produção elevada de resíduos sólidos, cuja ausência de segregação gera um alto custo considerando a coleta, o transporte e o destino final dos mesmos nasceu a ideia da proposição da parceria. Assim, por meio de ações conjuntas que visem à proteção do meio ambiente, com a sensibilização da comunidade interna, do Hospital Universitário Pedro Ernesto (HUPE) e da Faculdade de Enfermagem (ENF) propomos desenvolver ações em prol de uma gestão eficiente dos resíduos sólidos recicláveis gerados no desenvolvimento de suas atividades diárias e que impactam o meio ambiente.

Por outro lado há de se pensar em estratégias eficientes, tendo em vista o aumento do volume de resíduos descartados em aterros, lixões, entre outros, além da elevação dos custos e da falta de locais apropriados referentes à disposição final e também da dificuldade de se ter um local para o despejo desses resíduos de maneira segura e menos agressiva ao meio ambiente ${ }^{6}$.

Sendo assim, ao minimizar a produção dos resíduos comuns por meio da segregação daqueles passíveis de reciclagem, há a redução da quantidade disposta desses resíduos no meio ambiente?

Portanto, a proposta deste Projeto se baseia em ações simples, reais e possíveis de implementação, tendo em vista a minimização da geração de resíduos sólidos e da segregação de resíduos passíveis de reciclagem. Em vez de fim da linha, reentrada no ciclo produtivo; a criação de um programa de educação e conscientização da comunidade hospitalar, bem como a necessidade da Faculdade de Enfermagem se adequar às novas práticas e usos de resíduos; a criação de programa de Educação Ambiental, incluindo alunos da Universidade do Estado do Rio de Janeiro. Embora, como citamos acima, sejam ações simples, contudo, dependem do apoio da Direção do HUPE e da Faculdade de Enfermagem.

A partir destas considerações iniciais relacionamos os seguintes objetivos: relatar a experiência da implantação do projeto intitulado A Reciclagem na coleta de papel na Faculdade de Enfermagem e no Hospital Universitário Pedro Ernesto: uma prática de ensino, extensão e pesquisa nos setores administrativos do HUPE; promover ações educativas e capacitar multiplicadores para democratização do conhecimento desenvolvido.

A implantação desse projeto irá possibilitar à Faculdade de Enfermagem e ao HUPE atender ao Decreto ${ }^{7}$ número 40.645/2007 do Governo Estadual que institui a separação dos resíduos recicláveis descartados pelos órgãos e entidades da administração pública estadual direta e indireta na fonte geradora e a Resolução $14 / 2007^{8}$ da Secretaria de Estado do Ambiente que estabelece as normas para implantação do processo de coleta seletiva solidária. Além do mais, promover ações educativas para a formação e a qualificação dos discentes, dos docentes, dos técnicos, administrativos e do pessoal de Limpeza da Faculdade de Enfermagem e do HUPE este projeto trará benefícios como a redução do volume de resíduos a ser enviado para destinação final.

\section{Metodologia}

Trata-se de pesquisa descritiva, quantitativa, desenvolvida em 2010 no HUPE/UERJ, aprovada pelo Comitê de Ética em Pesquisa do Hospital Universitário Pedro Ernesto da Universidade do Estado do Rio de Janeiro, sob o protocolo $\mathrm{n}^{\circ}$ 2987/2011.

Para o desenvolvimento do projeto foram estabelecidas as seguintes etapas: seleção das unidades; realização de entrevistas e visitas nas unidades; avaliação do modelo de gestão de resíduos das unidades; identificação das fontes de geração ${ }^{9}$ de resíduos sólidos e recicláveis e dos tipos de lixeiras existentes; caracterização quantitativa da unidade 
para cálculo da quantidade de resíduo gerado no setor e estimativa da taxa de geração de resíduo; identificação das lixeiras; capacitação dos alunos, docentes, servidores e usuários dos ambientes selecionados, por meio de palestras e de oficinas.

As atividades começaram em maio de 2010, sendo inicialmente o HUPE/UERJ a primeira unidade de implantação da coleta seletiva. Optou-se pela implementação parcial nas unidades devido à necessidade de realização de cursos, treinamento da equipe e previsão de gastos para compra de materiais necessários. Desse modo, a primeira etapa iniciou nos setores administrativos do HUPE/UERJ de modo a favorecer a formação de uma cultura institucional em que o cidadão entenda sua parcela de responsabilidade diante dos problemas ambientais.

Para implantação do projeto nesse ambiente foi realizado um diagnóstico situacional avaliando primeiramente o quantitativo e os tipos de resíduos comuns que eram produzidos.

A partir do desenvolvimento do projeto, percebeu-se a potencial possibilidade de redução do volume de geração diária de resíduos comuns, o que reduz, ainda, os custos referentes à coleta, transporte e destino final dos mesmos. Ao pensar em estratégias e mecanismos de realização para essa redução, foi elaborado um questionário com os seguintes objetivos: traçar o perfil dos funcionários da área administrativa; avaliar seu conhecimento em relação à reciclagem e à sensibilização à proposta e dimensionar o quantitativo de lixeiras que, de acordo com eles, seriam necessárias para realizar a coleta do papel, apontado anteriormente pelos entrevistados como o resíduo reciclável mais gerado nestes setores.

Foram sujeitos da pesquisa 162 funcionários técnico-administrativos voluntários de ambos os sexos que demonstraram interesse em participar da pesquisa. Em 18 unidades do HUPE, foram aplicados 162 questionários com os participantes do estudo, foram respondidos 135 questionários cujos dados foram organizados, analisados e apresentados na modalidade de frequência simples e percentual.

\section{Resultados}

Dos 162 questionários, 135 (83\%) foram respondidos e os resultados relacionados ao per- fil do funcionário ficaram assim distribuídos: 79 (58\%) do grupo é do sexo feminino e 56 (42\%) do sexo masculino; no que se refere à modalidade de contrato de trabalho, 91 (67\%) são funcionários de contrato temporário, sendo 66 (49\%) de nível médio e 25 (18\%) de nível superior, enquanto que 29 (21\%) são servidores concursados, dos quais 18 (13\%) de nivel médio e 11(8\%) de nivel superior; com relação ao tempo de serviço no HUPE 39 (28\%) têm menos de 1 ano; 49 (36\%) têm de 1 a 3 anos, 12 (9\%) de 3 a 5 anos e $32(23 \%)$ mais de 5 anos.

Os resultados referentes aos questionamentos acerca do conhecimento e sensibilidade dos funcionários sobre a temática indicam que 119 (88\%) dos entrevistados consideram como muito importante o HUPE ter um gerenciamento de resíduos e 88 (65\%) referem saber que existe norma e órgãos reguladores para este gerenciamento; porém, 95 (70\%) dos participantes desconhecem os procedimentos básicos de segregar e acondicionar corretamente estes resíduos.

Ainda com relação ao conhecimento dos funcionários, os resultados apontam que 133 (93\%) entrevistados sabem o que é reciclagem, $112(83 \%)$ consideram a implantação de coleta para reciclagem no HUPE como muito importante, $113(84 \%)$ separaria os resíduos como forma de ajudar ao projeto, $61 \%(82)$ já coletou algum tipo de material para a reciclagem, 90\%(121) indicaram o papel impresso como o tipo de papel que o setor poderia separar para a reciclagem e $74 \%$ (100) não conhecem o destino final dos papéis coletados para a reciclagem.

Os dados demonstram que as pessoas ouvem muito o termo reciclagem, porém conhecem esse tema de maneira superficial e não se percebem responsáveis pela produção dos resíduos. Sobre a existência de um programa de gerenciamento de resíduos, $88,15 \%$ dos funcionários consideram muito importante e a maioria dos funcionários demonstrou interesse em contribuir direta ou indiretamente com as atividades propostas pelo projeto.

Além dos resultados do questionário, o grupo entendeu ser importante realizar um diagnóstico da situação da coleta do papel. Assim, evidenciou-se que em $80 \%$ dos setores o papel impresso estava misturado com outros tipos de resíduos, além de papéis de outras cores. Em 47\% dos setores todas as lixeiras para papel branco continham 
sacos plásticos. No que tange ao número de lixeiras por setor, os funcionários demonstraram que o número era insuficiente, uma vez que a proximidade da mesma à sua mesa é um fator facilitador da segregação correta.

A próxima etapa do projeto é a implementação na Faculdade de Enfermagem, seguindo os mesmos moldes, além da manutenção no HUPE. Estão previstas ações, a saber: planejamento de atividades de informação como palestras, distribuição de panfletos informativos, acompanhamento dos descartes que estão sendo realizados pelos funcionários e a solicitação da compra do quantitativo de lixeiras.

Quanto ao ambiente da Faculdade de Enfermagem, foram selecionados 29 setores nos 3 andares da unidade onde será implantada a coleta seletiva. As etapas subsequentes permeiam a aplicação dos questionários, sensibilização e treinamento das pessoas responsáveis pela limpeza dos andares da faculdade, bem como de docentes e discentes. É necessária, também, a análise e adequação de um espaço físico específico para a armazenagem adequada desse material que será segregado, a avaliação da parte de licença junto aos órgãos competentes para realização desta atividade, a análise de empresas que farão a coleta desse material e a necessidade de compra de balança para a medição da quantidade de papel que está sendo destinada à reciclagem.

Atualmente, na Faculdade de Enfermagem, foi realizado o levantamento dos setores nos quais serão iniciadas as seguintes atividades: aplicação do questionário e sensibilização e treinamento das pessoas responsáveis pela limpeza da $\mathrm{Fa}$ culdade de Enfermagem, bem como a análise e adequação de um espaço físico específico para a armazenagem desse material que será segregado, a avaliação da parte de licença junto aos órgãos competentes para realização desta atividade, a análise de empresas que farão a coleta desse material, além da necessidade de compra de balança para a medição da quantidade de papel que está sendo destinada à reciclagem.

\section{Conclusão}

O projeto encontra-se em desenvolvimento, sendo realizadas reuniões, atividades de levantamento e planejamento das ações no ambiente da
Faculdade de Enfermagem e do HUPE. Destaca-se que os objetivos do projeto vêm sendo alcançados no que tange às ações educativas para formação e a qualificação dos discentes, dos docentes, dos técnico-administrativos e das pessoas responsáveis pela limpeza, além da maior sensibilização do grupo para a coleta no HUPE, representado pela melhor qualidade e quantidade existente na segregação do papel.

Desta maneira, fica expressa a contribuição para a formação de multiplicadores junto à comunidade, o que engendra um efeito cascata no qual cada indivíduo se sente capacitado a abordar temáticas sobre a reciclagem em outros ambientes sociais, tornando-se cada vez mais ativos e participativos na disseminação desse conhecimento de extrema relevância para a perpetuação da sustentabilidade do planeta.

Merece destacar como contribuição do projeto, as boas práticas de gerenciamento de resíduos, envolvendo alunos da graduação o que vai caracterizar a sensibilização de futuros profissionais na gestão de resíduos. Por fim, ressalta-se que ainda há o trabalho de práticas que diminuem os danos causados ao meio ambiente, bem como a redução dos custos que seriam gerados à Faculdade de Enfermagem e ao HUPE em relação aos valores de coleta, transporte e destinação dos resíduos recicláveis que terminam sendo descartados como resíduos comuns e não destinados à reciclagem.

O projeto vem também utilizando estratégias que foram criadas com fins educativos por meio de filipetas, cartazes, jornais eletrônicos (Ecofolha), e-mail específico do projeto que são os canais de comunicação entre os sujeitos ativos e os organizadores, informando as atividades e andamento do projeto.

Garantimos a divulgação das ações do Projeto, a partir da sua participação na modalidade pôster, em 2010, em eventos de cunho acadêmico e de alcance da comunidade, como UERJ Sem Muros, Congresso Científico do HUPE, no IV Seminário de Enfermagem/ENFCUIDAR e no Congresso de Gerenciamento em Enfermagem.

A divulgação do projeto em eventos científicos vem contribuindo para que as ações desenvolvidas sejam ampliadas para diferentes públicos sensibilizando-os para a reflexão sobre o consumo consciente visto estarmos propondo a prática dos três Rs, a Reciclagem. 
A realização de cursos nos eventos como a Semana de Enfermagem (2010 e 2011) e no $48^{\circ}$ Congresso Científico do HUPE contribuem para a democratização do conhecimento.

\section{Contribuição dos autores}

Luciana Guimarães Assad - Concepção da ideia, elaboração do artigo e revisão geral.

Lina Márcia Miguéis Berardinelli - Concepção da ideia, elaboração do artigo e da estrutura textual, construção metodológica e finalização.

Dalila Passos Pereira da Silva - Elaboração do artigo, revisão da literatura, referências e conclusão.

Antônio Augusto Braga de Oliveira Junior - Levantamento bibliográfico e auxílio na coleta dos dados.

Thamires Goldim Rodrigues - Participação no levantamento da temática, coleta, organização dos dados e colaboração na análise.

\section{Referências}

1. SCHMIDHEINY, S. Mudando o Rumo: uma perspectiva empresarial global sobre desenvolvimento e meio ambiente. Rio de Janeiro: FGV, 1992. 368 p.

2. MATTAR, H. Consumidores Conscientes: O que pensam e como agem. Disponível em: www.akatu.org.br. Acesso em: 15 jun. 2011.

3. VINHA, V. G. A Convenção do Desenvolvimento Sustentável e As Empresas. Tese de Doutorado. Curso de Pós-Graduação em Desenvolvimento. Agricultura e Sociedade - CPDA/UFRRJ. Rio de Janeiro, 2000, 262 p.

4. SCHRAMM, F. R. Ecologia, ética e saúde: O principio da responsabilidade. In: Saúde, Ambiente e Desenvolvimento, p. 233-255, vol. 2, 1992. São Paulo: Hucitec/Rio de Janeiro: Abrasco.

5. TRIGUEIRO, A. Mundo Sustentável: abrindo espaço na mídia, para um planeta em transformação. São Paulo: Globo, 2005. 302 p.

6. BRASIL, M. S. ANVISA. Manual de Gerenciamento de Resíduos de Serviços de Saúde. Brasília: 2006. 182 p.

7. GUADAGNIN, M.R. Gerenciamento de Resíduos de Saúde: É possível minimizar? Disponível em: <www.unesc. net/noticias/index.php?ver=arquivo $>$. Acesso em: 17 mar. 2010.

8. BRASIL, M. S. Consumo Sustentável. Manual de educação, publicado pelo instituto Brasileiro de Defesa do consumidor (Idec) e pelo ministério do meio ambiente (MMA) em 2002.160 p.

9. BRASIL, M. S. ANVISA. Resolução de Diretoria Colegiada - RDC n ${ }^{\circ}$ 306, de 07 de dezembro de 2004. Disponível em: <http://www.unesp.br/pgr/pdf/rdc30604anvisa.pdf>. Acesso em: 30 jun. 2010.

\begin{abstract}
Report of an experience originated from the extension project, "Recycling in paper collection at the Nursing School and the Pedro Ernesto University Hospital (HUPE): a learning, extension and research practice". The aim is to report the experience of implementing the selective collection of paper at the administrative departments of Pedro Ernesto Hospital (HUPE) of the Rio de Janeiro State University and to present the results of a descriptive study that was undertaken at HUPE in 2010. 162 questionnaires were applied to HUPE's technical-administrative officers in 18 units. 135 questionnaires were returned, whose data were organized and chartered under simple and percentage frequencies. The results show that collecting solid waste for recycling is regarded as very important by $83 \%$ of the subjects. The project is implemented at HUPE in 18 administrative sectors and will also be implemented in 29 classrooms at the Nursing School of the Rio de Janeiro State University (UERJ). The project actions have contributed to the training of multipliers within the community and, above all, it fosters the daily reflection on the necessity of reducing, reusing and recycling the waste produced at partner units on a daily basis.
\end{abstract}

Keywords: Solid Waste; Environmental Education; Recycling 